\title{
More space for space
}

\author{
Born from astronomy, the study of planets is becoming increasingly geoscience. As divisions between \\ disciplines continue to blur in Solar System studies, at Nature Geoscience we are looking forward to \\ exciting joint projects with Nature Astronomy.
}

Today, rovers on Mars zap lasers and drill holes, and orbiters send back remote sensing data from the outer Solar System at high resolution. But planetary science was born from astronomy. Until the Soviet and American lunar programmes of the 1950s, our view of other planetary bodies was limited to telescopes. With exploration, the Solar System came into focus. Instead of coloured spheres of light tracked across the sky with telescopes, one by one each planet and moon was revealed by spacecraft to be a complex world shaped by geological processes. Ever since the data started to pour back, the skill set needed to understand these worlds has shifted away from astronomy expertise in geology, geophysics and atmospheric science is now in demand. The Solar System today is a playground for geoscientists seeking to understand how planets (including our own) work. Such advances in planetary geoscience continue to find a home in Nature Geoscience, although we look forward to the company of our sister journal, Nature Astronomy, which launches this month (http://www. nature.com/natastron/) and bridges to the astronomy roots of planetary science.

The past decade has seen spacecraft such as Dawn, Hayabusa and Rosetta visit asteroids and comets up close, and Cassini has revealed the geologic diversity in the Saturn system at high resolution. In 2015, our view of Pluto evolved from a pixelated point of light seen through telescopes, to a planet with a hazy atmosphere and complex surface geology when New Horizons flew past (Fig. 1). At Pluto - a body that has been demoted from classification as a planet New Horizons poignantly illustrated that planetary processes operate similarly on different bodies despite vastly different conditions, whether on Earth or in the frigid outermost Solar System (Schenk, P. \& Nimmo, F. Nat. Geosci. 9, 411-412; 2016).

Planetary astronomy is no longer the prominent component of observatory programmes that it once was. Nevertheless, it remains an active field of planetary science, especially when it comes to asteroids, comets and exoplanets. In

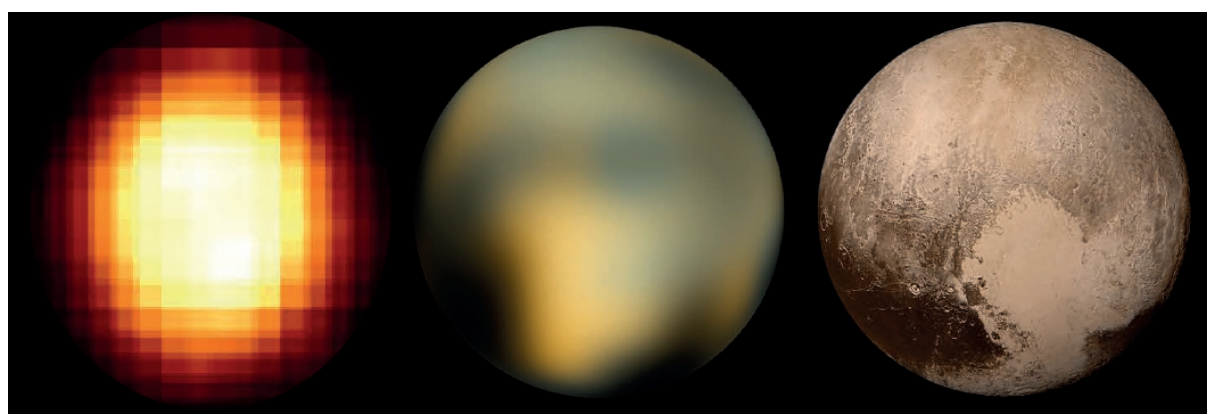

Figure 1 | Pluto coming into focus. Pluto as seen from a ground-based telescope (left; Gemini Observatory/ NSF/NASA/AURA), the Hubble Space Telescope (centre; NASA, ESA and M. Buie of the Southwest Research Institute) and the New Horizons flyby (right; NASA/JHUAPL/Southwest Research Institute).

addition, the pursuit to understand how our Solar System fits into the larger view of the formation and evolution of stellar systems, galaxies and the universe remains in the remit of astronomy.

Planetary science has become increasingly multidisciplinary to accommodate the many types of observational data, as well as the laboratory and numerical analyses that go along with figuring out what those observations mean. The broad scope of planetary science is reflected in the diverse educational backgrounds of planetary scientists. Some come from geoscience backgrounds, others from astronomy or astrophysics, or one of the other scientific disciplines.

We are pleased to welcome Nature Astronomy to the family of Nature journals. Nature Astronomy is an online-only journal covering astronomy, astrophysics and - like Nature Geoscience - planetary science. Together, Nature Geoscience and Nature Astronomy will span the full spectrum of planetary sciences from their most geoscience- to astronomy-based research, and everything in between. The most important message for the planetary science community: there will be more space for space in the Nature Research Group's portfolio of journals.

As for all Nature journals, Nature Geoscience and Nature Astronomy are editorially independent. That is, editors of one journal do not have access to another journal's database, papers or referee information. Nevertheless we look forward to working with our sister journals to showcase planetary science research from across the Nature family.

If you are lucky enough to have a piece of research that could fit equally well into Nature Geoscience and Nature Astronomy which is the case for most planetary science studies - consider which audience you are most keen to address. Nature Geoscience aims to appeal to readers from across the Earth and planetary sciences, whereas Nature Astronomy mostly addresses readers from space science communities.

If you think your paper will be of interest to Earth scientists and thus would benefit from the extra visibility a Nature Geoscience paper would bring to that community, then you might consider submitting to us. If you think your paper would benefit from extra visibility to the astronomy community, then you might consider Nature Astronomy. And of course, if one journal declines, you are welcome to submit to the other. Not that we would ever let that influence our decision, but since the journals are editorially independent, we will not even know that we were not your first choice, unless you tell us yourself.

Understanding how planets form and how they work - whether Earth or a distant world, and whether your tool is a telescope, spacecraft, computer or laboratory - are quests that often involve geoscience. We look forward to continuing the planetary journey with our readers. 